\title{
Defibrillation time intervals and outcomes of cardiac arrest in hospital: retrospective cohort study from Get With The Guidelines- Resuscitation registry
}

\author{
Steven M Bradley, ,2,3 Wenhui Liu, ${ }^{1,4}$ Paul S Chan, ${ }^{1}$ Brahmajee K Nallamothu, 6 Gary K Grunwald,,,4 \\ Alyssa Self, ${ }^{2}$ Comilla Sasson, ${ }^{7}$ Paul D Varosy, ${ }^{1,2,3}$ Monique L Anderson, ${ }^{8}$ Preston M Schneider, ${ }^{2,3}$ \\ P Michael Ho ${ }^{1,2,3}$ for the American Heart Association's Get With The Guidelines-Resuscitation \\ Investigators
}

\section{ABSTRACT}

OBJECTIVE

To describe temporal trends in the time interval between first and second attempts at defibrillation and the association between this time interval and outcomes in patients with persistent ventricular tachycardia or ventricular fibrillation (VT/VF) arrest in hospital.

\section{DESIGN}

Retrospective cohort study.

\section{SETTING}

172 hospitals in the United States participating in the Get With The Guidelines-Resuscitation registry, 2004-12. PARTICIPANTS

Adults who received a second defibrillation attempt for persistent VT/VF arrest within three minutes of a first attempt.

\section{INTERVENTIONS}

Second defibrillation attempts categorized as early (time interval of up to and including one minute between first and second defibrillation attempts) or deferred (time interval of more than one minute between first and second defibrillation attempts).

\section{MAIN OUTCOME MEASURE}

Survival to hospital discharge.

\section{RESULTS}

Among 2733 patients with persistent VT/VF after the first defibrillation attempt, 1121 (41\%) received a deferred second attempt. Deferred second defibrillation for persistent VT/VF increased from $26 \%$ in 2004 to $57 \%$ in 2012 ( $P<0.001$ for trend). Compared with early second defibrillation, unadjusted patient outcomes were significantly worse with deferred second defibrillation $(57.4 \% \vee 62.5 \%$ for return of

\section{WHAT IS ALREADY KNOWN ON THIS TOPIC}

Resuscitation guidelines were revised in 2005 to recommend single shock protocols with two minutes of chest compressions between defibrillation attempts for persistent ventricular tachycardia/ventricular fibrillation (VT/VF)

Resuscitation guidelines for VT/VF were primarily informed by studies of cardiac arrest outside hospital and might not reflect optimal management of VT/VF arrest in hospital

\section{WHAT THIS PAPER ADDS}

Consistent with the adoption of resuscitation guidelines, the proportion of patients with a deferred second attempt at defibrillation (more than one minute between attempts) doubled from about $25 \%$ in 2004 to slightly more than $50 \%$ in 2012 Deferred second defibrillation was not associated with improved patient outcomes for those with VT/VF in hospital spontaneous circulation, $38.4 \%$ v $43.6 \%$ for survival to 24 hours, and $24.7 \%$ v $30.8 \%$ for survival to hospital discharge; $\mathrm{P}<0.01$ for all comparisons). After risk adjustment, deferred second defibrillation was not associated with survival to hospital discharge (propensity weighting adjusted risk ratio $0.89,95 \%$ confidence interval 0.78 to $1.01 ; \mathrm{P}=0.08$; hierarchical regression adjusted $0.92,0.83$ to $1.02 ; \mathrm{P}=0.1)$.

\section{CONCLUSIONS}

Since 2004, the use of deferred second defibrillation for persistent VT/VF in hospital has doubled. Deferred second defibrillation was not associated with improved survival.

\section{Introduction}

Defibrillation is critical in the resuscitation of patients with cardiac arrest due to ventricular tachycardia or ventricular fibrillation (VT/VF). Resuscitation guidelines have previously called for up to three successive or "stacked" shocks with minimal time delays between defibrillation attempts. ${ }^{1}$ The use of stacked shocks, however, can result in prolonged interruptions of chest compressions that can contribute to poor patient outcomes. ${ }^{2-5}$ As a result, in 2005 resuscitation guidelines were revised to support single shock protocols with two minutes of chest compressions between defibrillation attempts to minimize interruptions in compressions. ${ }^{6-8}$

Several studies of cardiac arrest outside hospital support the current resuscitation guidelines for the management of VT/VF with single shock protocols. ${ }^{9-11}$ A prior randomized trial comparing a stacked versus single shock protocol in the management of cardiac arrest outside hospital, however, found no difference in patients' outcomes. ${ }^{12}$ Furthermore, the characteristics of VT/VF arrest in hospital differ from outside hospital in ways that can influence the relative importance of rhythm termination versus chest compressions. Compared with patients with VT/VF outside hospital, patients with VT/VF in hospital are often monitored or witnessed at the time of arrest, resulting in rapid recognition of arrest and shorter times to initiation of chest compressions and first attempt at defibrillation. ${ }^{13}$ As a result, they are treated in the earlier phases of cardiac arrest, when an emphasis on rhythm termination with shorter intervals between defibrillation attempts might improve outcomes. ${ }^{14}$ More frequent defibrillation attempts, however, might increase interruptions of chest compressions and subsequently contribute to poor patient outcomes. ${ }^{15}$ Data on the duration of time between defibrillation attempts in response to changes in 
resuscitation guidelines and its effect on survival for patients with cardiac arrest in hospital are lacking.

Understanding of the relation of timing of second defibrillation attempt for those with VT/VF in hospital has implications for the 40000 patients who experience VT/VF in hospital each year. ${ }^{16} 17$ Using data from a large national quality improvement registry of cardiac arrest in hospital, we first examined temporal trends in the time interval between first and second attempts at defibrillation among patients with persistent VT/VF after the first attempt. We anticipated that the time interval between attempts would have increased in the past decade, consistent with adoption of single shock protocols in the management of VT/VF in hospital. We then examined the association between the time interval from first to second attempts at defibrillation and survival of patients to hospital discharge. As data on the timing of second attempts in relation to patients' outcomes are currently lacking for persistent VT/VF in hospital, any findings should provide empirical evidence to inform future resuscitation guidelines.

\section{Methods}

\section{Study setting and design}

We used data from the American Heart Association's (AHA) Get With The Guidelines-Resuscitation registry (GWTG-R). This is a large national prospective multicenter data registry and quality improvement program for cardiac arrest in hospital. Details regarding the registry design, data collection, and quality oversight have been described elsewhere. ${ }^{13}$ Briefly, participating hospitals in the United States voluntarily report data on all patients with a confirmed cardiac arrest (defined by the lack of a palpable central pulse, apnea, and unresponsiveness) who received cardiopulmonary resuscitation. Participating hospitals use multiple approaches to case finding to ensure complete capture of all cases of cardiac arrest in hospital, including review of cardiac arrest flow sheets and the hospital paging system logs, routine checks of code carts (carts stocked with emergency drugs and equipment) and pharmacy tracer drug records, and hospital billing charges for use of resuscitation drugs. ${ }^{17}$ At each facility, trained nurses/research coordinators abstract data from medical records and code review sheets using Utstein definitions for cardiac arrest in hospital. ${ }^{18}$ Accuracy of abstracted data is facilitated through certification of data entry personnel, data checks for missing or outlying observations, case study methods for newly enrolled hospitals prior to data submission, and periodic re-abstraction process. A random audit of the re-abstraction process showed a mean error rate of $2.4 \%$ (SD $2.7 \%$ ) for all data. ${ }^{13}$ The AHA provides quality control and oversight for GWTG-R data collection, analysis, reporting, and research studies.

\section{Patient population}

We identified 30500 patients aged 18 or older who received defibrillation for an index cardiac arrest in hospital in which the first identifiable rhythm was ventricular fibrillation or pulseless ventricular tachycardia at 615 hospitals participating in GWTG-R in 2004-12.
Among patients with multiple cardiac arrests during the same hospital admission, we included only the first event in the analysis. We excluded 1487 (4.9\%) patients who received only one defibrillation attempt during the resuscitation event and $21283(69.8 \%)$ patients who were no longer in VT/VF after the first attempt (that is, the rhythm immediately after defibrillation was not VT/ VF). Accordingly, our study also excludes patients with recurrent VT/VF as the time interval between defibrillation attempts in these patients is defined by the time of recurrent VT/VF, rather than the provider's treatment decision immediately after failed defibrillation.

Our eligible cohort therefore comprised 7730 patients who received a second defibrillation attempt for persistent VT/VF after an initial defibrillation attempt. From this cohort, we excluded 2764 (35.8\%) patients in whom the time of the first or second defibrillation attempt was unknown. Comparison of standardized differences between patients with known and unknown defibrillation intervals were less than 5\% for nearly all characteristics patients and arrests, suggesting that patients with available data on defibrillation time intervals were representative of the overall patient population (appendix, fig A). We excluded 154 (2.0\%) patients with missing data on covariates used in risk adjusted analyses (that is, pre-existing conditions or interventions in place at the time of the intervention). We also excluded 1429 (18.5\%) patients whose time from first to second defibrillation attempt was more than three minutes, given concerns that this prolonged interval was inconsistent with guidelines and could represent unusual resuscitation circumstances that could not be determined from the registry data. We further restricted our final analytic cohort to the 2733 patients at the 172 hospitals with adequate sample size (six or more eligible events) as we sought to examine outcomes by defibrillation intervals within hospitals (fig 1).

\section{Patient involvement}

No patients were involved in setting the research question or the outcome measures, nor were they involved in

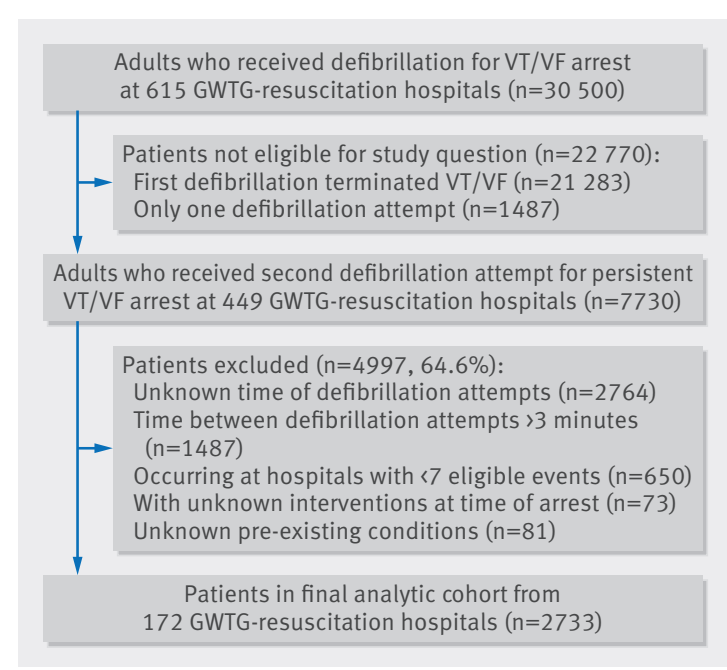

Fig 1 | Flow and selection of patients in study of defibrillation time intervals and outcomes of cardiac arrest in hospital 
developing plans for design or implementation of the study. No patients were asked to advise on interpretation or writing up of results. There are no plans to disseminate the results of the research to study participants or the relevant patient community. Results will be disseminated to hospitals participating in the GWTG-R through the AHA.

\section{Time interval between defibrillation attempts}

Defibrillation time within GWTG-R is captured in one minute intervals. Time between defibrillation attempts was calculated as the interval from the reported time of first attempt to the reported time of the second attempt. Both reported times were recorded in minutes and determined from documentation of cardiac arrest in the patient's medical records. As these data are captured from documentation of routine practice at hospitals participating in GWTG-R, the modality of time capture is not standardized across facilities. In our primary analysis, we used these data to determine proportion of patients in whom the second defibrillation attempt was early (defined as a time of a minute or less between first and second defibrillation) or deferred (defined as more than a minute between defibrillation attempts). As the time of defibrillation attempts in GWTG-R is recorded in minutes, we chose this categorization to reflect stacked shocks with minimal time delays between defibrillation attempts compared with single shock protocols with a period of chest compressions between defibrillation attempts. In secondary analyses, we classified patients according to whether the time between first and second defibrillation attempts was less than a minute, one minute, two minutes, or three minutes.

\section{Study outcomes}

Our primary outcome was survival to hospital discharge. Secondary outcomes included return of spontaneous circulation for at least 20 minutes after onset of the arrest, survival to 24 hours, and neurological status among those patients who survived to discharge as determined by cerebral performance category score (1=mild or no neurologic disability, $2=$ moderate neurologic disability, $3=$ severe neurologic disability, 4=coma or vegetative state, and $5=$ brain death). We dichotomized scores to assess for no major neurologic disability (score $\leq 2$ at discharge) in relation to defibrillation time intervals.

\section{Statistical analysis}

We first evaluated temporal trends in the time between defibrillation attempts by calendar year with linear regression. We then compared characteristics of patients and arrests at baseline by early and deferred second defibrillation attempts using non-parametric Kolmogorov Smirnov test for continuous variables and $\chi^{2}$ squared for categorical variables.

Our risk adjusted analysis used a multilevel propensity score weighting model, which accounts for the propensity to receive deferred defibrillation across hospitals. We determined this propensity from a non-parsimonious logistic regression model that included 39 patient level covariates and accounted for hospital variation in the propensity for deferred second defibrillation attempts (appendix, table A). ${ }^{19}$ From the propensity score model, we calculated the stabilized inverse probability weight. We evaluated balance in the patients' covariates using standardized differences of the covariates between the early and deferred second defibrillation groups. After weighting, the maximum standardized difference was $7 \%$, with most under $2.5 \%$, much smaller than the commonly accepted adequate balance of $10 \%$ (appendix, fig B). ${ }^{20}$ The outcome model included the main effect for the defibrillation time interval, weighted by the stabilized inverse probability weight. The sandwich estimator of the variance was used in estimating the standard error of the estimated main effect, to account for clustering of patients within hospitals and for modified Poisson estimation of relative risk. We then performed survival analyses using Kaplan-Meier curves and Cox model weighted on the inverse probability of treatment weights. Additional details of the propensity score weighted analysis are provided in the appendix.

As a sensitivity analysis, we used hierarchical modified Poisson regression, ${ }^{21}$ which uses a robust sandwich estimator to account for clustered observations by hospital and determined the risk adjusted association between deferred second defibrillation for persistent VT/VF and patient outcomes. Based on prior literature, our models adjusted for patient demographics (age, sex, race), diagnosis on admission (medical, cardiac; medical, non-cardiac; surgical, cardiac; surgical, non-cardiac; obstetric; trauma; other), pre-existing disorders at the time of arrest (acute stroke, baseline depression in central nervous system, hepatic insufficiency, hypotension, major trauma, metastatic cancer, septicemia, myocardial infarction this admission, none), therapeutic interventions in place at the time of cardiac arrest (assisted ventilation, invasive airway device, chest tube, monitoring with an arterial line, antiarrhythmic drugs, vasopressor, vasodilators), characteristics of the cardiac arrest (witnessed arrest, arrest location (intensive care unit, monitored unit, non-monitored unit, procedural area, other), time of arrest (daytime, night time, weekend), time to first defibrillation attempt), and calendar year. ${ }^{2223} \mathrm{~A}$ full list of the variable specifications for covariates used in the multivariable models is provided in appendix table A.

Statistical analyses were performed with SAS/STAT software, version 9.4 of the SAS System for Windows and all statistical tests were evaluated $\mathrm{P} \leq 0.05$.

\section{Results}

During the study period, the proportion of patients with deferred second defibrillation attempts increased from $26 \%$ (18/68) in 2004 to 57\% (59/103) in 2012 ( $\mathrm{P}<0.001$ for trend) (fig 2). In secondary analyses categorized by one minute intervals, there was a decrease in the proportion of patients with less than a minute between defibrillation attempts, from $31 \%$ (21/68) in 2004 to $8 \%$ (8/103) in 2012 (appendix, fig C). Over the same time period there was an increase in the proportion of patients with two 


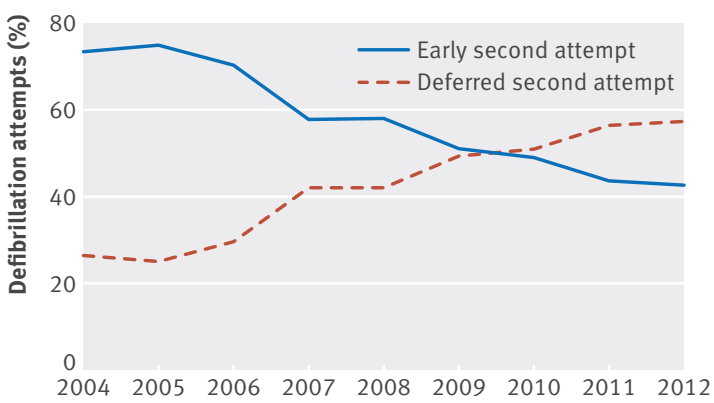

Year

Fig 2 | Temporal trends in early (one minute or less) and deferred (more than one minute) defibrillation intervals in patients with cardiac arrest in hospital

minutes between defibrillation attempts from 22\% (15/68) in 2004 to 33\% (34/103) in 2012 and a similar increase in the proportion with three minutes between defibrillation attempts from $4 \%$ (3/68) in 2004 to $24 \%$ $(25 / 103)$ in 2012. Exploratory analyses showed an increase in the proportion excluded from the cohort because of more than three minutes between defibrillation attempts $(14.7 \%(22 / 150)$ in 2004 to $24.3 \%$ (111/457) in 2012), consistent with a temporal increase in time between defibrillation attempts (appendix, fig D).

Among 2733 patients with persistent VT/VF after the first defibrillation attempt, $1121(41.0 \%)$ received a deferred second defibrillation attempt. Compared with patients who received an early second defibrillation attempt, patients with a deferred second attempts were less likely to have hypotension ( 20.3 v 25.6\%, $\mathrm{P}=0.001)$, experience myocardial infarct during the current admission (24.6 $v 29.0 \%, \mathrm{P}=0.01)$, and more likely to have septicemia $(14.7 \% \vee 9.9 \%, \mathrm{P}<0.001)$. Time from onset of arrest to first defibrillation was longer in patients with deferred second defibrillation attempts (median time to defibrillation one versus two minutes, $\mathrm{P}<0.001)$ and patients with deferred second attempts were more likely to be in a non-monitored unit $(11.2 \% \mathrm{~V}$ 9.1\%, $\mathrm{P}<.001)$ and less likely to be in procedural areas $(26.8 \% v 73.2 \%, \mathrm{P}<0.001)$ (table 1$)$.

Achievement of return of spontaneous circulation occurred in 1651 patients (60.4\%), while 1131 patients (41.4\%) survived to 24 hours after cardiac arrest, and 772 (27.8\%) survived to hospital discharge. Compared with early second defibrillation attempts, unadjusted patient outcomes were significantly worse after deferred second defibrillation attempts $(57.4 \% v 62.5 \%$ for return of spontaneous circulation, $38.4 \% v 43.6 \%$ for survival to 24 hours, and $24.7 \% v 30.8 \%$ for survival to hospital discharge; $\mathrm{P}<0.01$ for all comparisons). In risk adjusted analyses with propensity weighting methods for risk adjustment, a deferred second defibrillation attempt was not associated with survival to hospital discharge (adjusted risk ratio $0.89,95 \%$ confidence interval 0.78 to 1.01; $\mathrm{P}=0.08$ ). Similar results were observed in survival analyses (appendix, fig E). With hierarchical regression, a deferred second defibrillation attempt was not associated with survival to hospital discharge (0.92, 0.83 to 1.02 ; $\mathrm{P}=0.12$ ). After risk adjustment, deferred defibrillation was also not associated with return of spontaneous circulation or survival at 24 hours. Among patients who survived to hospital discharge, we observed similar findings for the association between deferred second defibrillation and the likelihood of having severe neurologic disability (propensity weighting adjusted risk ratio $0.94,0.84$ to 1.06 ) (table 2).

\section{Discussion}

In a large US national registry of cardiac arrest in hospital, we sought to describe temporal trends in time intervals between defibrillation attempts for persistent ventricular tachycardia or ventricular fibrillation (VT/ $\mathrm{VF}$ ) arrest and to determine the association between the timing of a second defibrillation attempt and patient outcomes. From 2004 to 2012, the proportion of patients with a deferred second defibrillation attempt (more than one minute between defibrillation attempts) doubled from about $25 \%$ to slightly more than $50 \%$. Deferred second defibrillation, however, was not associated with improved patient outcomes. These findings stand in contrast to current resuscitation guidelines that emphasize two minutes of chest compressions between defibrillation attempts as optimal care for persistent VT/VF in hospital. ${ }^{8}$

Contemporary resuscitation guidelines were revised in 2005 on the basis of animal models and observational studies that showed the detrimental impact of prolonged interruptions in chest compressions resulting from stacked shocks in rapid succession. ${ }^{2-4}$ These prior studies, however, primarily reflect resuscitation outside hospital, including delays between onset of VT/VF and initiation of chest compressions and first defibrillation attempt. For example, animal model studies that supported single shock protocols evaluated initiation of resuscitation care after several minutes without cardiopulmonary resuscitation. ${ }^{23-25}$ Human studies supporting the use of single shock defibrillation protocols are from settings outside hospital, ${ }^{910}$ in which nearly half of arrests are unwitnessed with average delays of several minutes between recognition of arrest, initiation of cardiopulmonary resuscitation, and attempted defibrillation. ${ }^{26}$ Whether the benefit of single shock protocols extends to settings in hospital, where cardiac arrests are often witnessed or monitored, initiation of cardiopulmonary resuscitation occurs rapidly, and time to first defibrillation is short, has not been previously explored. ${ }^{13}$ In fact, European Resuscitation Council guidelines suggest that stacked shocks can be considered for cardiac arrests occurring in certain witnessed or procedural settings. ${ }^{27} 28$ This guideline provision is consistent with a prior conceptual model of cardiac arrest that emphasizes the importance of defibrillation for rhythm termination in the early temporal phases of cardiac arrest. ${ }^{14}$ The findings from the current study agree with this conceptual model and suggest that greater emphasis on rhythm termination for persistent VT/VF might be warranted in hospital.

Notably, in our study, VT/VF was terminated with the first defibrillation attempt in under $70 \%$ of patients. This contrasts with prior small studies that have reported efficacy of first shock of $>90 \%$ with VT/VF outside 


\begin{tabular}{|c|c|c|c|}
\hline \multirow[b]{2}{*}{ Characteristic } & \multicolumn{2}{|c|}{ Second defibrillation attempt } & \multirow[b]{2}{*}{ Pvalue } \\
\hline & $\begin{array}{l}\text { Early }(\leq 1 \mathrm{~min} \\
\text { between attempts) } \\
(\mathrm{n}=1612,59 \%)\end{array}$ & $\begin{array}{l}\text { Deferred }(>1 \mathrm{~min} \\
\text { between attempts) } \\
(n=1121,41 \%)\end{array}$ & \\
\hline Mean (SD) age (years) & $64.22(15.2)$ & $65.22(14.7)$ & 0.09 \\
\hline Men & $1059(65.7)$ & $733(65.4)$ & 0.9 \\
\hline White & $1209(75.0)$ & $820(73.2)$ & 0.7 \\
\hline \multicolumn{4}{|l|}{ Pre-existing conditions: } \\
\hline Heart failure this admission & $277(17.2)$ & $193(17.2)$ & 0.9 \\
\hline Previous heart failure & $307(19.0)$ & $243(21.7)$ & 0.09 \\
\hline Myocardial infarction this admission & $467(29.0)$ & $276(24.6)$ & 0.01 \\
\hline Previous myocardial infarction & $328(20.4)$ & $237(21.1)$ & 0.6 \\
\hline Arrhythmia & $660(40.9)$ & $448(40.0)$ & 0.6 \\
\hline Hypotension & $413(25.6)$ & $227(20.3)$ & 0.001 \\
\hline Respiratory insufficiency & $559(34.7)$ & $376(33.5)$ & 0.5 \\
\hline Hepatic insufficiency & $86(5.3)$ & $60(5.4)$ & 0.9 \\
\hline Metabolic or electrolyte abnormality & $197(12.2)$ & $153(13.7)$ & 0.3 \\
\hline Diabetes mellitus & $459(28.5)$ & $350(31.2)$ & 0.1 \\
\hline Baseline depression in CNS function & $170(10.6)$ & $105(9.4)$ & 0.3 \\
\hline Acute stroke & $50(3.1)$ & $44(3.9)$ & 0.3 \\
\hline Pneumonia & $109(6.8)$ & $88(7.9)$ & 0.3 \\
\hline Septicemia & $159(9.9)$ & $165(14.7)$ & $<0.001$ \\
\hline Major trauma & $55(3.4)$ & $33(2.9)$ & 0.5 \\
\hline Metastatic cancer & $124(7.7)$ & $109(9.7)$ & 0.06 \\
\hline \multicolumn{4}{|l|}{ Interventions in place before arrest: } \\
\hline Mechanical ventilation & $592(36.7)$ & $368(32.8)$ & 0.04 \\
\hline Intravenous vasopressor medication & $549(34.1)$ & $327(29.2)$ & 0.007 \\
\hline Dialysis & $47(2.9)$ & $41(3.7)$ & 0.3 \\
\hline Intra-aortic balloon pump & $72(4.5)$ & $31(2.8)$ & 0.02 \\
\hline Pulmonary artery catheter & $105(6.5)$ & $49(4.4)$ & 0.02 \\
\hline \multicolumn{4}{|l|}{ Characteristics of cardiac arrest } \\
\hline \multicolumn{4}{|l|}{ Initial cardiac arrest rhythm: } \\
\hline Ventricular fibrillation & $1029(63.8)$ & $741(66.1)$ & \multirow{2}{*}{0.2} \\
\hline Pulseless ventricular tachycardia & $583(36.2)$ & $380(33.9)$ & \\
\hline Median time (mins) to first defibrillation attempt (IQR) & $1(0-4)$ & $2(0-5)$ & $<0.001$ \\
\hline \multicolumn{4}{|l|}{ Hospital location of arrest: } \\
\hline Intensive care unit & $418(25.9)$ & $343(30.6)$ & \multirow{4}{*}{$<0.001$} \\
\hline Monitored unit & $168(10.4)$ & $190(17.0)$ & \\
\hline Non-monitored unit & $146(9.1)$ & $126(11.2)$ & \\
\hline Procedural area & $265(73.2)$ & $97(26.8)$ & \\
\hline Hospital-wide response activated & $1141(70.8)$ & $817(72.9)$ & 0.2 \\
\hline Assessed with AED & $287(17.8)$ & $248(22.1)$ & 0.01 \\
\hline \multicolumn{4}{|l|}{ Time of arrest: } \\
\hline Arrest at night (11 pm to $7 \mathrm{am})$ & $283(17.6)$ & $247(22.0)$ & \multirow{2}{*}{0.01} \\
\hline Arrest on weekend & $429(26.6)$ & $275(24.5)$ & \\
\hline \multicolumn{4}{|l|}{ Hospital characteristics } \\
\hline \multicolumn{4}{|l|}{ Geographic region: } \\
\hline North and Mid-Atlantic & $231(14.3)$ & $161(14.4)$ & \multirow{5}{*}{0.7} \\
\hline South Atlantic & $403(25.0)$ & $268(23.9)$ & \\
\hline North central & $369(22.9)$ & $243(21.7)$ & \\
\hline South central & $287(17.8)$ & $224(20.0)$ & \\
\hline Mountain/Pacific & $286(17.7)$ & $196(17.5)$ & \\
\hline \multicolumn{4}{|l|}{ Hospital size (No of beds): } \\
\hline$<250$ & $229(14.2)$ & $154(13.7)$ & \multirow{3}{*}{0.3} \\
\hline $250-499$ & $741(46.0)$ & $463(41.3)$ & \\
\hline$\geq 500$ & $606(37.6)$ & $475(42.4)$ & \\
\hline
\end{tabular}

$A E D=$ automated external defibrillator, $C N S=$ central nervous system, IQR=interquartile range.

hospital..$^{29-33}$ As a result of these prior studies, guideline writing committees have raised concerns about the incremental benefit of an immediate second defibrillation attempt relative to resumption of chest compres- sions. ${ }^{67}$ Our study suggests persistent VT/VF is pertinent for a larger proportion of VT/VF in hospital than would be expected from prior studies of cardiac arrest outside hospital. As a result, understanding the appropriate 


\begin{tabular}{|c|c|c|c|c|c|}
\hline \multirow[b]{2}{*}{ Outcome } & \multicolumn{2}{|c|}{ No (\%) with second defibrillation attempt } & \multicolumn{3}{|l|}{ Risk ratio $(95 \% \mathrm{CI})$} \\
\hline & Early & Deferred & Unadjusted & $\begin{array}{l}\text { Propensity } \\
\text { weighted adjusted }\end{array}$ & $\begin{array}{l}\text { Hierarchical } \\
\text { regression adjusted }\end{array}$ \\
\hline \multicolumn{6}{|l|}{ Survival outcomes } \\
\hline Return of spontaneous circulation & $1008 / 1612(62.5)$ & $643 / 1121(57.4)$ & 0.92 (0.86 to 0.98$)$ & 0.94 (0.88 to 1.01$)$ & $0.95(0.90$ to 1.01$)$ \\
\hline Survival to 24 hours & $701 / 1606(43.6)$ & $430 / 1121(38.4)$ & $0.88(0.80$ to 0.96$)$ & 0.93 (0.84 to 1.03$)$ & $0.95(0.87$ to 1.04$)$ \\
\hline Survival to discharge & $495 / 1605(30.8)$ & $277 / 1121(24.7)$ & 0.80 (0.71 to 0.91) & 0.89 (0.78 to 1.01) & $0.92(0.83$ to 1.02$)$ \\
\hline \multicolumn{6}{|c|}{ Neurologic outcome (survivors only) } \\
\hline No major disability & $306 / 443(69.1)$ & $152 / 236(64.4)$ & $0.93(0.83$ to 1.04$)$ & $0.94(0.83$ to 1.06$)$ & 0.98 (0.88 to 1.10$)$ \\
\hline
\end{tabular}

balance between defibrillation for rhythm termination and minimization of interruptions to chest compression could have even greater implications for in hospital.

Despite a lack of data on single shock defibrillation protocols in hospital, we observed a significant temporal increase in the proportion of deferred second defibrillation attempts from 2004 to 2012. This is consistent with adoption of 2005 resuscitation guidelines and greater use of single shock protocols with a period of chest compressions between defibrillation attempts. Several studies from settings outside hospital have shown improvement in patient outcomes with adoption of guideline changes, which included single shock protocols for defibrillation of VT/VF. ${ }^{10} 11$ A randomized trial that compared stacked and single shock protocols outside hospital, however, failed to show improved patient outcomes with single shock defibrillation. ${ }^{12}$

Overall temporal trends in survival after VT/VF arrest in hospital have improved in the past decade and could reflect the impact of changes in resuscitation care in accordance with resuscitation guidelines. ${ }^{17}$ It is important to note, however, that guideline revisions were not restricted to defibrillation protocols but also highlighted minimizing interruptions in chest compression for the placement of invasive airways or invasive lines and the importance of avoiding excessive ventilation. ${ }^{67}$ Our findings do not conflict with the benefit of these guideline recommendations overall but raise questions about the specific benefit of deferred second defibrillation attempts for VT/VF in hospital.

\section{Strengths and limitations}

Strengths of the current study include the use of national multicenter data from a well established registry of cardiac arrest in hospital with high data quality. Our results, however, should also be considered in light of the following limitations. Although we used multiple methods for risk adjustment in the comparison of outcomes by defibrillation interval, we cannot exclude the possibility of unmeasured confounding in this observational study. Second, in the registry the time interval between defibrillation attempts is captured in minutes, and this limits the ability to evaluate the association between defibrillation time intervals and patient outcomes by smaller units of time. Furthermore, we cannot exclude misclassification of time intervals related to challenges of exact documentation of event times during care of patients who have cardiac arrest in hospital; although, we would expect this to bias our results towards the null in the absence of differential misclassification. Third, we lacked data on other aspects of quality of cardiopulmonary resuscitation, including frequency and duration of chest compressions or compression rate, depth, and hands-off time-factors known to correlate with patient outcomes. Fourth, as arrests in hospital in our study were often monitored or witnessed, our findings might be less applicable to health systems with lower use of inpatient monitoring and longer times to recognition of arrest. Similarly, the association between defibrillation intervals and patient outcomes might be influenced more by the time to arrest recognition and treatment than the event setting (that is, in hospital versus outside hospital). We were, however, unable to identify patients with delayed recognition of VT/VF arrest within the registry to further explore this relation. Finally, we lacked data on whether stacked or single shock defibrillation protocols were used in the care of these registry patients and instead used the time interval between first and second defibrillation attempts as our measure of defibrillation practice. The increasing prevalence of deferred second defibrillation after publication of the 2005 resuscitation guidelines suggests these time intervals are a reasonable measure of defibrillation practice.

\section{Conclusion}

The time interval between defibrillation attempts for persistent VT/VF in hospital has increased in the past decade. These findings are consistent with adoption of resuscitation guidelines that support single shock protocols with chest compressions between defibrillation attempts. Deferred defibrillation for persistent VT/VF, however, was not associated with improved survival. Further study is necessary to understand whether current guidelines, which recommend against immediate second defibrillation attempts for persistent VT/VF in hospital, need reconsideration.

\section{Get With The Guidelines-Resuscitation Adult Task Force}

SMB, PSC, Saket Girotra (University of lowa Carver College of Medicine); Michael W Donnino (Beth Israel Deaconess Medical Center); Dana P Edelson (University of Chicago); Robert T Faillace (Geisinger Healthcare System); Romergryko Geocadin (Johns Hopkins University School of Medicine); Raina Merchant (University of Pennsylvania School of Medicine); Vincent N Mosesso (University of Pittsburgh School of Medicine); Joseph P Ornato and Mary Ann Peberdy (Virginia Commonwealth University).

Contributors: SMB was responsible for conception, design, analysis, data interpretation, and manuscript writing. WL and GKG analyzed and interpreted data and wrote the manuscript. PSC, BKN, and PMH designed the study, interpreted data, and wrote the manuscript. AS, CS, PDV, MLA, and PMS interpreted data and wrote the manuscript. SMB had full access to all data in the study and takes responsibility for the integrity of the data and the accuracy of the data analysis and is guarantor.

Funding: This research received no specific grant from any funding agency in the public, commercial, or not-for-profit sectors. SMB is 
supported by a career development award (HSR\&D-CDA2 10-199) from VA Health Services Research and Development. The views expressed in this article are those of the authors and do not necessarily reflect the position or policy of the Department of Veterans Affairs or the US government.

Competing interest: All authors have completed the ICMJE uniform disclosure form at www.icmje.org/coi_disclosure.pdf and declare: no support from any organization for the submitted work; no financial relationships with any organizations that might have an interest in the submitted work in the previous three years; no other relationships or activities that could appear to have influenced the submitted work.

Ethical approval: This study was approved by the Colorado multiple institutional review board.

Data sharing: No additional data available. Requests for GWTG-R data are managed by the American Heart Association (http://www.heart. org/HEARTORG/HealthcareResearch/Healthcare-Research_ UCM_001093_SubHomePage.jsp).

Transparency: The lead author affirms that this manuscript is an honest, accurate and transparent account of the study being reported; that no important aspects of the study have been omitted; and that any discrepancies from the study as planned (and, if relevant) have been explained.

This is an Open Access article distributed in accordance with the Creative Commons Attribution Non Commercial (CC BY-NC 3.0) license, which permits others to distribute, remix, adapt, build upon this work non-commercially, and license their derivative works on different terms, provided the original work is properly cited and the use is non-commercial. See: http://creativecommons.org/licenses/ by-nc/3.0/.

1 Guidelines 2000 for Cardiopulmonary Resuscitation and Emergency Cardiovascular Care. Part 6: advanced cardiovascular life support: section 2: defibrillation. The American Heart Association in collaboration with the International Liaison Committee on Resuscitation. Circulation 2000:102(Suppl):190-4

2 Yu T, Weil MH, Tang W, et al. Adverse outcomes of interrupted precordial compression during automated defibrillation. Circulation 2002;106:368-72. doi:10.1161/01.CIR.0000021429.22005.2E.

3 Kern KB, Hilwig RW, Berg RA, Sanders AB, Ewy GA. Importance of continuous chest compressions during cardiopulmonary resuscitation: improved outcome during a simulated single lay-rescuer scenario. Circulation 2002;105:645-9. doi:10.1161/hc0502.102963.

4 Eftestøl T, Sunde K, Steen PA. Effects of interrupting precordial compressions on the calculated probability of defibrillation success during out-of-hospital cardiac arrest. Circulation 2002;105:2270-3. doi:10.1161/01.CIR.0000016362.42586.FE.

5 Berg RA, Sanders AB, Kern KB, et al. Adverse hemodynamic effects of interrupting chest compressions for rescue breathing during cardiopulmonary resuscitation for ventricular fibrillation cardiac arrest. Circulation 2001;104:2465-70. doi:10.1161/hc4501.098926.

6 ECC Committee, Subcommittees and Task Forces of the American Heart Association. 2005 American Heart Association Guidelines for Cardiopulmonary Resuscitation and Emergency Cardiovascular Care. Circulation 2005;112(Suppl):IV1-203.

7 Link MS, Atkins DL, Passman RS, et al. Part 6: electrical therapies: automated external defibrillators, defibrillation, cardioversion, and pacing: 2010 American Heart Association Guidelines for Cardiopulmonary Resuscitation and Emergency Cardiovascular Care. Circulation 2010;122(Suppl 3):S706-19. doi:10.1161/ CIRCULATIONAHA.110.970954

8 Link MS, Berkow LC, Kudenchuk PJ, et al. Part 7: Adult Advanced Cardiovascular Life Support: 2015 American Heart Association Guidelines Update for Cardiopulmonary Resuscitation and Emergency Cardiovascular Care. Circulation 2015;132(Suppl 2):S444-64. doi:10.1161/CIR.0000000000000261.

9 Bobrow BJ, Clark LL, Ewy GA, et al. Minimally interrupted cardiac resuscitation by emergency medical services for out-of-hospital cardiac arrest. JAMA 2008;299:1158-65. doi:10.1001/jama.299.10.1158.

10 Rea TD, Helbock M, Perry S, et al. Increasing use of cardiopulmonary resuscitation during out-of-hospital ventricular fibrillation arrest: survival implications of guideline changes. Circulation 2006;114:2760-5. doi:10.1161/CIRCULATIONAHA.106.654715.

11 Hinchey PR, Myers JB, Lewis R, et al. Capital County Research Consortium. Improved out-of-hospital cardiac arrest survival after the sequential implementation of 2005 AHA guidelines for compressions, ventilations, and induced hypothermia: the Wake County experience. Ann Emerg Med 2010;56:348-57. doi:10.1016/j. annemergmed.2010.01.036.

12 Jost D, Degrange H, Verret C, et al. DEFI 2005 Work Group. DEFI 2005 : a randomized controlled trial of the effect of automated external defibrillator cardiopulmonary resuscitation protocol on outcome from out-of-hospital cardiac arrest. Circulation 2010;121:1614-22. doi:10.1161/CIRCULATIONAHA.109.878389.
13 Peberdy MA, Kaye W, Ornato JP, et al. Cardiopulmonary resuscitation of adults in the hospital: a report of 14720 cardiac arrests from the National Registry of Cardiopulmonary Resuscitation. Resuscitation 2003;58:297-308. doi:10.1016/S0300-9572(03)00215-6.

14 Weisfeldt ML, Becker LB. Resuscitation after cardiac arrest: a 3-phase time-sensitive model. JAMA 2002;288:3035-8. doi:10.1001/ jama.288.23.3035.

15 Abella BS, Alvarado JP, Myklebust H, et al. Quality of cardiopulmonary resuscitation during in-hospital cardiac arrest. JAMA 2005;293:30510. doi:10.1001/jama.293.3.305.

16 Merchant RM, Yang L, Becker LB, et al. American Heart Association Get With The Guidelines-Resuscitation Investigators. Incidence of treated cardiac arrest in hospitalized patients in the United States. Crit Care Med 2011;39:2401-6. doi:10.1097/CCM.0b013e3182257459.

17 Girotra S, Nallamothu BK, Spertus JA, Li Y, Krumholz HM, Chan PS. American Heart Association Get with the Guidelines-Resuscitation Investigators. Trends in survival after in-hospital cardiac arrest. N Engl J Med 2012;367:1912-20. doi:10.1056/NEJMoa1109148.

18 Cummins RO, Chamberlain D, Hazinski MF, et al. American Heart Association. Recommended guidelines for reviewing, reporting, and conducting research on in-hospital resuscitation: the in-hospital 'Utstein style'. Circulation 1997;95:2213-39. doi:10.1161/01.CIR.95.8.2213.

19 Li F, Zaslavsky AM, Landrum MB. Propensity score weighting with multilevel data. Stat Med 2013:32:3373-87. doi:10.1002/sim.5786.

20 Normand ST, Landrum MB, Guadagnoli E, et al. Validating recommendations for coronary angiography following acute myocardial infarction in the elderly: a matched analysis using propensity scores. J Clin Epidemiol 2001;54:387-98. doi:10.1016/S0895-4356(00)00321-8.

21 Zou GY, Donner A. Extension of the modified Poisson regression model to prospective studies with correlated binary data. Stat Methods Med Res 2013:22:661-70. doi:10.1177/0962280211427759.

22 Larkin GL, Copes WS, Nathanson BH, Kaye W. Pre-resuscitation factors associated with mortality in 49,130 cases of in-hospital cardiac arrest: a report from the National Registry for Cardiopulmonary Resuscitation. Resuscitation 2010;81:302-11. doi:10.1016/j.resuscitation.2009.11.021.

23 Chan PS, Berg RA, Spertus JA, et al. AHA GWTG-Resuscitation Investigators. Risk-standardizing survival for in-hospital cardiac arrest to facilitate hospital comparisons. J Am Coll Cardiol 2013;62:601-9. doi:10.1016/j.jacc.2013.05.051.

24 Cammarata G, Weil MH, Csapoczi P, Sun S, Tang W. Challenging the rationale of three sequential shocks for defibrillation. Resuscitation 2006;69:23-7. doi:10.1016/j.resuscitation.2005.09.022

25 Tang W, Snyder D, Wang J, et al. One-shock versus three-shock defibrillation protocol significantly improves outcome in a porcine model of prolonged ventricular fibrillation cardiac arrest. Circulation 2006;113:2683-9. doi:10.1161/CIRCULATIONAHA.105.592121.

26 Nichol G, Thomas E, Callaway CW, et al. Resuscitation Outcomes Consortium Investigators. Regional variation in out-of-hospital cardiac arrest incidence and outcome. JAMA 2008;300:1423-31. doi:10.1001/ jama.300.12.1423.

27 Nolan JP, Soar J, Zideman DA, et al. ERC Guidelines Writing Group. European Resuscitation Council Guidelines for Resuscitation 2010 Section 1. Executive summary. Resuscitation 2010;81:1219-76. doi:10.1016/j.resuscitation.2010.08.021

28 Monsieurs KG, Nolan JP, Bossaert LL, et al. ERC Guidelines 2015 Writing Group. European Resuscitation Council Guidelines for Resuscitation 2015: Section 1. Executive summary. Resuscitation 2015:95:1-80. doi:10.1016/j. resuscitation.2015.07.038.

29 Poole JE, White RD, Kanz KG, et al. LIFE Investigators. Low-energy impedance-compensating biphasic waveforms terminate ventricular fibrillation at high rates in victims of out-of-hospital cardiac arrest. J Cardiovasc Electrophysiol 1997;8:1373-85. doi:10.1111/j.1540-8167.1997.tb01034.x.

30 White RD, Blackwell TH, Russell JK, Snyder DE, Jorgenson DB. Transthoracic impedance does not affect defibrillation, resuscitation or survival in patients with out-of-hospital cardiac arrest treated with a non-escalating biphasic waveform defibrillator. Resuscitation 2005:64:63-9. doi:10.1016/j.resuscitation.2004.06.021.

31 Mittal S, Ayati S, Stein KM, et al. ZOLL Investigators. Comparison of a novel rectilinear biphasic waveform with a damped sine wave monophasic waveform for transthoracic ventricular defibrillation. J Am Coll Cardiol 1999:34:1595-601 doi:10.1016/S0735-1097(99)00363-0.

32 Schneider T, Martens PR, Paschen H, et al. Optimized Response to Cardiac Arrest (ORCA) Investigators. Multicenter, randomized, controlled trial of 150-J biphasic shocks compared with 200- to 360-J monophasic shocks in the resuscitation of out-of-hospital cardiac arrest victims. Circulation 2000;102:1780-7. doi:10.1161/01.CIR.102.15.1780.

33 Morrison LJ, Henry RM, Ku V, Nolan JP, Morley P, Deakin CD. Single-shock defibrillation success in adult cardiac arrest: a systematic review. Resuscitation 2013;84:1480-6. doi:10.1016/j. resuscitation.2013.07.008.

Appendix: Supplementary figures, text, and table 